\title{
BLADDER DRAINAGE AND PROSTATISM
}

\author{
By STePhen Power, M.S., F.R.C.S.
}

Senior Surgeon, Dreadnought Hospital; Surgeon, Eltham and Nottingham Hospital; Surgeon, London Homoeopathic Hospital $\overrightarrow{\overrightarrow{\text { Pे }}}$

Since the bladder cannot be drained by any route for more than a day or two without infecting it, and infection accounts for most of the morbidity and mortality of prostatectomy, drainage is a matter that deserves the most serious attention. It is unfortunate that as often as not its management is automatically relegated to a junior, inexperienced though he may be. An acute retention should be regarded as an emergency of the same order as a perforation or appendicitis; although the patient may not go to the operating theatre at once, he certainly needs the services of the surgical registrar in the ward.

The "aseptic" school of prostatectomists do, of course, take him straight to the theatre, and so avoid pre-operative drainage altogether. Even at the time of operation they will only permit retrograde catheterization, so that there shall be no possibility of organisms lurking in the anterior urethra being carried into the bladder on the catheter tip. But these surgeons are forced to employ drainage for several days after operation and, in consequence, their figures for subsequent infection may be no different from any others. Their enthusiasm has, however, helped to emphasize the vital importance of avoiding preliminary drainage if this is at all possible, and minimizing it, if it is not, so that the operation is conducted in a sterile or near-sterile field and nature is given an opportunity of laying down a barrier of granulation tissue before infection really sets in. It must be mentioned that one patient in five is already infected when he first sees a doctor, though the organism is usually the relatively innocuous $B$. Coli.

The urinary tract is itself something of a drainage system, working more or less according to the ordinary laws of hydrostatics, especially when the bladder is deprived of its contractility and transformed into an inert bag by some form of catheterization. Dependent drainage now takes on increased importance, not only for the bladder but for the kidneys as well. It is significant that, when paraplegic patients are allowed up and about, the urinary infection from which they invariably suffer always improves. In them, of course, reflux up the ureter is quite common, and the erect position must discourage it very considerably. $\stackrel{\mathbb{2}}{\varrho}$ But even in the healthy subject, the pelves of the kidneys drain much better in the erect position $\overrightarrow{0}$ than the prone, a fact that can be demonstratedquite dramatically by sitting a patient up for $\vec{\rho}$ a second or two during a retrograde pyelography.ర

Case note. A patient had been in bed under 3 medical treatment for six weeks. His prostate was only slightly enlarged, but after using a bottle for so long his bladder harboured a residual of $20 \mathrm{oz}$. The urine had never shown more than a few leucocytes. He could not produce a specimen in bed at the time of the examination, but ono standing up beside the bed passed one which was so thick with pus that it looked like soup. Later, $\subseteq$ at cystoscopy, a purulent mass was seen occupying the bladder base, where it would have remained $\overrightarrow{0}$ as long as the patient stayed in bed. Pus sinks foll the bottom of the bladder in exactly the same way that it sinks to the bottom of a specimen glass.

Case note. A patient had been on daily bladder washes for some time. One day the house surgeon. reported that he had emptied an abscess. In fact, $\stackrel{\circ}{\mathbb{Q}}$ he had readjusted the catheter so that the eye lay just inside the meatus instead of an inch or two응 higher up. A catheter inserted too far does not drain the base of the bladder; if its tip impinges? on the fundus it may produce an ulcer or even a perforation.

\section{Catheter Drainage}

The simplest and most obvious method of drainage is by passing a catheter up the urethra. Provided this is done with proper aseptic pre-o cautions and with reasonable gentleness and skill, $\rightarrow$ there is much to be said in its favour as a means o of relieving an attack of acute retention. But if $\underset{N}{\sim}$ the procedure has to be carried out more than $\sigma$ once, the risk of introducing infection increases. $N$ enormously; repeated catheterization makes it N almost impossible to maintain sterility. Such ${ }^{\omega}$ infection is likely to prove particularly dangerous if the kidneys have already been damaged by back $\frac{0}{\Phi}$ pressure. So it should never be resorted to for $\stackrel{?}{?}$ the purpose of emptying a chronically distended $T$ bladder outside the four walls of a hospital. This condition being painless, or relatively painless, 
there is fortunately never any need to treat it on the spot.

Even in acute retention, catheterization is not free from danger, largely because of the circumstances in which the attack occurs. Asepsis must often be imperfect, the distressed state of the patient forbids any unnecessary delay, while his fruitless efforts to relieve himself by squeezing out even a drop of urine only, serve to increase the muscular spasm and render the passage of a catheter more difficult than ever.

In spite of the urgency, removal of the patient to hospital is still the best course. When this is impossible, he should be given an injection of morphia. If it is given intravenously, relief is not long delayed, and there is now an opportunity for cleaning the parts and making what preparations are necessary to maintain asepsis.

A local anaesthetic should certainly be used. The late Edward Canny Ryall, founder of All Saints' Hospital, in his atlas of cystoscopy wrote, - The humblest patient has the same claim as the millionaire in this respect, an equal right to be spared suffering.' Canny Ryall used a solution of one-half per cent. cocaine and sodium bicarbonate, which gives excellent results. But it deteriorates in solution and has to be freshly prepared each time it is used and it should never be used after instrumentation. Xylocaine jelly (Duncan \& Flockhart) is almost as effective, has the advantage of acting as a lubricant, and is ready for immediate use when the nozzle is fitted to the collapsible tube; Io c.c. of the jelly should be injected into the urethra in two lots, the first being massaged into the posterior urethra before the second is injected. The urethra is then clamped and 10 or $15 \mathrm{~min}$. allowed for anaesthesia to develop.

The choice of catheter is largely a personal matter. Prostatic enlargement does not narrow the urethra-in its posterior part its capacity is actually increased-so there is no need to choose a small catheter. One of the middle range which is not too rigid will be safest; the rigid variety is apt to go astray, and it is much more likely to do this in the anterior urethra than the posterior. In nine cases out of ten the accident occurs in the bulb. Here the channel widens, changes direction, and then closes down again as it passes through the triangular ligament between the fibres of the compressor muscle. Some skill is needed in keeping the tip of the advancing catheter on the right path and judging the exact amount of pressure required to overcome the resistance of this muscle. Any force exerted in a wrong direction produces a false passage. Progress is then halted and, when the catheter is withdrawn, blood drips or flows from the meatus. Not uncommonly the diagnosis is altered to one of stricture, and smaller

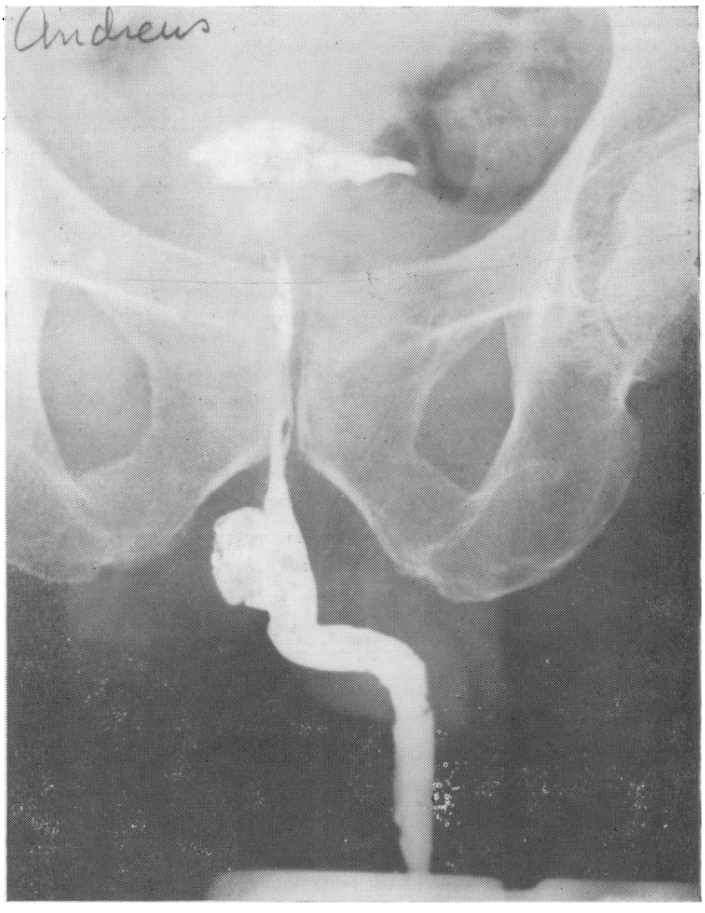

Urethrogram showing false passage.

and smaller catheters are tried without success. If the truth is realized it may be possible by hugging the roof of the urethra to reach the bladder, but the cul-de-sac often defeats every effort to by-pass it and may remain open for weeks or months, or perhaps become a permanent feature in the anatomy of the part.

It is because of this difficulty in the bulb and not because of the middle lobe that a Tieman's or a Coude catheter is so useful Once past the compressor the support of an assistant's finger placed per rectum, just below the apex of the prostate, may prevent further accidents.

One catheterization rarely cures prostatic retention. It must usually be repeated and, even then, it is only the retention that has been precipitated by some special circumstance, such as drinking too much beer or lying in bed after an operation, that is curable. If the retention arises for no particular reason it is likely to persist, in more or less degree. Even when the patient commences to pass water naturally again, the chances are he is only partly emptying his bladder and, if he has been catheterized several times, the residuum is probably infected and certain to remain so.

A catheter en demeure will certainly infect the bladder and usually with cocci as well as coli. But this is not the only risk. The urethra itself is studded with glands, the prostate drains into it, 
and the ejaculatory ducts put it into direct communication with the seminal vesicles and the epididymes. Urethritis is unavoidable, but vesiculitis may follow, and perhaps epididymitis as well. Even with this, the possibilities are not exhausted. Our predecessors were familiar with a condition which they called catheter fever; we recognize it as a form of septicaemia.

Case note. A soldier patient with stricture required regular dilatation. Each time he went back to his unit after treatment he reported sick. It was decided to admit him to hospital for 24 hours to see what was happening. After the next dilatation his temperature went up to 103 and $B$. Coli was grown from the blood.

I recollect another case in which a staphylococcus was recovered from the blood and a metastatic abscess formed in the arm of a patient who had been clumsily catheterized.

Septicaemia sequelae are commoner than we think. If we cross-questioned our stricture patients I have no doubt that quite a few of them would admit to having had a 'chill' on the night after dilatation, especially when the dilatation was followed by a little haemorrhage.

The anterior urethra traverses a blood sinus known as the corpus spongiosum, and the two are separated by no more than the urethral wall, a barrier that is easily bruised and more frequently breached than is generally realized. It is a salutary exercise to split open the penis and examine the urethra in all post-mortems where a catheter has been recently employed.

It is reported that during urethrography for stricture a patient collapsed, complaining of abdominal pain. $\mathrm{X}$-ray showed barium in the pelvic veins, which later reached the liver and spleen. Nine years after, the spleen was still opaque. Opportunities have arisen of demonstrating barium in the coronary and cerebral arteries under similar circumstances.

The not unknown death from embolism during air urethroscopy is another proof of the close association between the urethra and the circulatory system. Res ipsa loquitur.

Of course, there is another side to the picture. We have all heard of the elderly gentleman who carries on happily year after year passing a catheter on himself, which he keeps coiled up inside the crown of his hat and lubricates with a little spittle. $\mathrm{He}$ is not always elderly either. The British Medical Fournal some time ago published an account of an undergraduate who was left with an overflow retention following an injury to his spine. $\mathrm{He}$ started with his catheter when he was still under twenty and continued with it for fifty years or more until he died of old age. These stories are not apocryphal. Many of them are well authen- ticated, and they go to show that in the last resorts? the human body defends itself against infection by developing an immunity-local as well aso systemic. The urologist sees less dramatic examples of the same phenomenon every day in? the week; without it his mortality figures would be formidable.

\section{The Indwelling Catheter}

When the antibiotics were first introduced it was hoped that catheter urethritis would becomen a thing of the past, but this has not happened The continuing irritation of the catheter chafing against the mucous membrane still produces a continuing infection. Sometimes it almost seems $\overrightarrow{-}$ as if antibiotics do more harm than good.

Case note. The catheter was removed on the fourth day after a retropubic prostatectomy streptomycin being used prophylactically. At now time was there any clouding of the urine or stickiness of the meatus, but the patient com-8 plained of a general tenseness of the glans andew shaft of the penis. He returned soon after discharge from hospital with a pronounced narrowing of the whole urethra.

Although urethritis must be accepted when anc indwelling catheter is used, much may be done to minimize its severity and avoid its sequelae. $\overrightarrow{0}$

I. Manufacturers have only recently interest\&્đ themselves in the important question of the reactions that take place between human tissưos and materials brought into contact with them, and there is still much work to be done before theseo problems are settled, but there can be no question $\frac{0}{2}$ that the types of catheter put on the market during the last few years are much superior to the old ones. An indwelling catheter should be con-structed of a smooth and durable substance which does not crack or corrode, it should be flexible so as to accommodate itself to the curves of the 3 . urethra, and it should be as thin walled as possible, in order to give the maximum inside diameter. with the minimum outside. Also it should beo boilable. In sharp contrast to gum elastic, plastic $₹$ enjoys all these advantages. Latex can lay claim? to many of them and, in addition, seems to be $>$ better tolerated by the urethra than ordinary․ㅡ․ rubber, but like the Foley, it is collapsible, thoughñ this does not matter very much unless there is a need for the aspiration of clot. The Foley is $N$ superior to other catheters for comfort and cleanliness, but for anatomical reasons does noto drain as well before operation as after. It is aso well to make sure that the balloon empties as easily as it fills before using it; contretemps have. ${ }^{\text {? }}$ been recorded. Over-distension is unnecessary and may be dangerous; $10 \mathrm{mls}$. of fluid is enough $\overrightarrow{+}$ to keep the catheter in position; further stretching? 


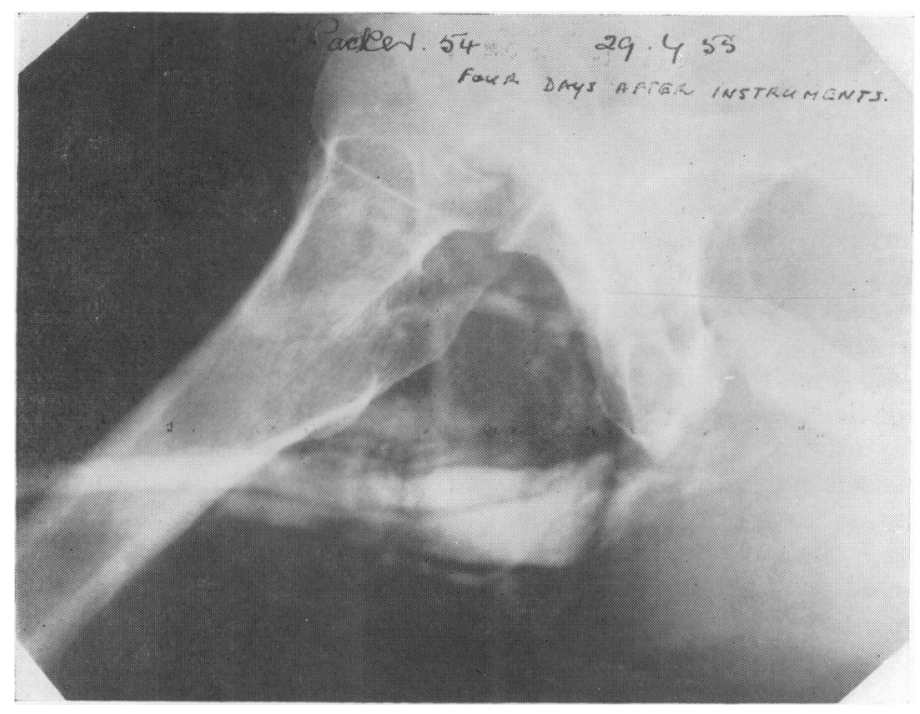

Urethrogram several days after instrumentation. The medium has escaped into the corpus spongiosum and adjacent vessels.

of the prostatic bed may lead to necrosis of the rectum. Care is needed to see that orientation is correct with the balloon and its inlet tube lying posteriorly; otherwise the tip may become kinked.

The Bardex catheter is of the Foley type, but less collapsible, although made of synthetic rubber; owing to exchange restrictions it is not obtainable in this country, but is very popular in America.

Catheters should be hollow tipped, so that an introducer can be used when required, and should have two lateral holes, so that if one lies against the mucous membrane of the bladder the other is free.

2. Whatever catheter is chosen should be loosely fitting so that discharge can escape freely alongside it. Nothing is so likely to provoke stricture formation as a tight fit with damming back of secretion; this is much more important than the length of time the catheter is kept in. Measurement has shown that the anterior urethra may be twice as wide in one man as another, so no hard and fast rules can be laid down, but the size of the penis can be taken as a rough guide to the size of the urethra, and the feel of the catheter as it is introduced should confirm that it is a comfortable fit. An $\mathrm{r} 8 \mathrm{~F}$ is nearly always safe; a size smaller than this may be sufficient to drain urine, but will not allow the escape of clots after operation.

3. The external meatus deserves particular attention since it is the narrowest part of the canal, and not unlikely to become the seat of stricture. Even a well-chosen catheter may be held up here during insertion if it has not been generously lubricated. When this detail has been attended to and there is still a suggestion of tightness a little novocaine should be injected and the meatus slit well back to one side of the fraenum. Not only does this get over the immediate difficulty but it ensures drainage later on.

4. When a catheter is to remain in the urethra it is more than ever important that no loophole should be created at the time of its introduction, for the escape of infected material into the corpus spongiosum, where, even if it did no more serious mischief, it might lead to peri-urethral infiltration with subsequent fibrosis; it is the spongy tissue surrounding the anterior urethra that renders it so much more prone to stricture formation than the posterior. En passant, one wonders how many of the multiple strictures still seen in our clinics are due to the instrumentation and not to the original infection.

5. With the catheter safely in position, the question arises of how it is to be fixed if it is not self-retaining. When strapping is used it must not be allowed to constrict the penis, or all the care taken over the fit may be wasted. Tight strapping here has been known not only to compress the urethra, but to lead to actual sloughing of its floor. A catheter should always be connected up to a jar at the side of the bed by a length of tubing. If its end is allowed to lie in a bottle placed between the patient's thighs, its contents may be sucked back into the bladder when the bottle fills. 
6. It is the house surgeon's duty on his twice daily visit to see that this whole area is kept scrupulously clean, and particularly that the meatus does not get gummed up by discharge. A patient with an indwelling catheter is apt to develop an insidious paraphimosis, which adds to his discomforts without causing actual pain. It may escape notice and become difficult to correct if the routine of inspection breaks down.

7. Finally, catheter drainage should be discontinued at the earliest possible moment. If it seems likely that it will be needed for more than a few days it is better eschewed altogether and supra-pubic drainage used from the start: if it has been started and some form of drainage must be continued then the switch over from one to the other must not be delayed. Failing this, an early vasectomy under local anaesthesia may save a lot of heart burning.

\section{Supra-pubic Drainage}

No method of fixing an indwelling catheter is completely reliable; even a Foley bag is not leakproof and, when it does leak, the catheter immediately slips out. Now, one of the rules for bladder drainage is that once begun it should be continued until there is no longer any need for it. To infect a bladder and then allow it to fill up again before the obstruction is relieved is asking for trouble. So the slipping out of a catheter may be a serious matter, unless it is replaced without delay.

From this point of view supra-pubic drainage is more satisfactory than catheter drainage and, in addition, it avoids all the local hazards of the urethra. But it infects the bladder just as readily as a catheter, and it offends against one of the elementary principles of all drainage-it is not dependent. It is, in fact, uphill. Nevertheless, it proves remarkably satisfactory even in the business of keeping the bladder empty, as can be seen on the urogram of any patient who has had a cystotomy. There are two reasons for this. In the first place the bladder collapses. In the second place in its collapsed state it is subject to the positive pressure of the abdominal viscera, which compress it into the pelvis and force its contents to the surface. Some surgeons consider continuous suction a necessary adjunct to this type of drainage, but in most cases such negative pressure is superfluous; nature's positive pressure is all that is required.

Supra-pubic drainage in favourable circumstances can be established with little more disturbance-sometimes considerably less-than the passage of a catheter. When the bladder is distended, so that it is easily identified in the hypogastrium, a tube can be inserted with a Riches introducer under local anaesthesia without so much as moving the patient from his bed. But? it is not safe to do this blindly. The bladder should be deliberately exposed through a small incision in order to make sure that the peritoneum is clear. After insertion the tube must be advanced ${ }^{\varrho}$ towards the base of the bladder; otherwise it will slip out as the viscus collapses. If the skinos incision is made halfway between the umbilicuso and the pubis, it will leave a long track and dis-등 courage vesical contracture; it will also give watertight drainage, and the rate at which urine $\mathbb{\Omega}^{\mathbb{\Phi}}$ escapes can easily be controlled, which is moreo than can be said for other methods of cystotomy. $\rightarrow$ Moreover, the operation field for a subsequent: prostatectomy is left almost untouched. There is, $\vec{\omega}$ however, the disadvantage that the Riches tube is and must be of small calibre. Should a heavyo infection ensue, with an alkaline urine throwing down phosphates, trouble is to be expected fromw blockage of the lumen. It may then be advisablew to change to a wider-bore tube.

The general rule can be laid down that if bladder drainage is needed for a few days $a_{0}^{N}$ catheter will do the job quite effectively, if for? a few weeks a Riches tube is excellent, but if for $_{\vec{C}}$ a few months an ordinary de Pezzer will give less $\stackrel{\complement}{<}$ trouble.

It takes some little time for a satisfactogyc $\overrightarrow{0}$ fistula to form after a cystotomy, so the ture. should not be changed for at least three week. If it stops draining it may be blocked and can cleared with a syringe; or it may have slippedō out of the bladder, although still held by theö recti, when it must be reintroduced. This is not足 always easy, and can be dangerous if $(a)$ the $\overrightarrow{0}$ cystotomy is less than ten days old or $(b)$ replace- 3 ment is delayed. In either case the alignment of the track may be lost, and the only safe way of: avoiding the peritoneum is to make a fresh punc-o음 ture after allowing the bladder to refill.

A patient with a cystotomy, whose vasa have $\frac{\sigma}{3}$ not been divided, should be warned not to micturate; if he attempts to do so a reflux of infected urine may cause an epididymitis.

\section{One- or Two-stage}

A man often dies of another disease than that which brought him to bed, so it is the part of $N$ wisdom to keep him out of it as much as possible. $\mathrm{N}$ No means of doing this after he has been admitted to hospital have yet been discovered. All time- $\omega$ consuming investigations should, therefore, be $\bar{\sigma}$ carried out while he is still an out-patient.

For similar reasons a one-stage prostatectomy has everything to be said for it. Unnecessarily to subject an elderly patient, or for that matter, $\frac{0}{0}$ any patient, to two operations instead of one, toत्त all the unpleasantness of supra-pubic drainage, $\frac{\stackrel{?}{\mathrm{C}}}{\mathrm{O}}$ 
accompanied by urinary infection, and to a prolonged illness followed by a delayed convalescence, is not good surgery and must increase the incidental mortality almost as much as it does the morbidity.

But there are times when even the enthusiasts hesitate to remove the prostate in one stage. Before coming to a decision the surgeon will remember that drainage means infection, that the infection continues as long as the drainage is maintained, that the longer the drainage is maintained the more difficult it will be to eradicate the infection, that if the drainage is maintained long enough the infection will become incurable, and that all this time the patient is liable to infective complications of various kinds, both local and remote. All this, notwithstanding drainage, may be required:

I. For the sake of the patient. The elderly prostate may be and often is hypertensive, arteriosclerotic, bronchitic, anaemic or diabetic; sometimes he suffers from a combination of these disorders. If his retention is treated by immediate prostatectomy there is very little time for diagnosing such complicating conditions, and none at all for treating them. There can be no doubt that a week of two in bed is beneficial to a man with a weakened myocardium.

2. For the sake of the kidneys. Symptoms and signs of uraemia, per se, are not a contra-indication to prostatectomy, so long as they are of recent onset and back pressure has not caused serious damage to the kidneys. But here a difficulty arises. How is it possible to ascertain the extent of the mischief? The tests, including intravenous pyelography, usually give the answer, but there is a less scientific, though often more reliable method of assessing the patient's chancesclinical judgment, taking into account not only his renal status but also his general health. Not that the two are unrelated. Uraemia affects every system in the body, and if the uraemia can be relieved every system reaps the benefit: which suggests the advisability of temporizing in a doubtful case by using an indwelling catheter in the hope that it will produce sufficient improvement to make prostatectomy safe without preliminary cystotomy.

Sometimes even prolonged drainage fails to relieve back pressure on the kidneys. This may be because the actual cause of the back pressure is not the intra-vesical tension but the hypertrophied vesical muscle. The combination of a thick-walləd bladder emptying reasonably well with markedly hydronephrotic kidneys is familiar enough. Drainage will do little good here, but it is indisputable that in the general run of cases it is followed by an improvement in the state of the kidneys.
3. For the sake of the bladder. The chronically distended bladder takes a long time to regain its tone. Two or three weeks drainage before operation gives it a chance to recover before it is called on to function again. Sometimes tone is permanently lost, and with a really atonic bladder in an elderly subject it is a question whether it is not better to leave well alone, since the patient will still have a residuum after operation, which will remain infected for the rest of his life.

\section{Decompression}

One writer has characterized the abrupt emptying of a chronically distended bladder as " calamitous catheterisation." Anyone who has seen the torrential haemorrhage that sometimes follows this procedure must agree with the description. A patient in reasonably good health (for a large residual may cause little or no upset so long as it is left alone) may be at death's door within 24 hours from shock, exsanguination, and anuria.

There is no detrusor hypertrophy to protect the ureters and pelves in these cases, so that a sudden fall in intra-vesical pressure produces an equally sudden fall of pressure in the pelves of the kidneys. Post-mortem blood and clot are found choking the whole urinary tract.

Some bleeding almost invariably follows the relief of a chronic retention, but bleeding of this order can be avoided by slow decompression. Two arguments are urged against this practice. First it is said that the haemorrhage is due not to the fall in pressure but to the introduction of infection. No one would deny that urinary infection is sometimes responsible for haematuria, but that it should cause a disaster of such magnitude seems scarcely credible. The prostatic himself may be grossly infected, and yet show no blood in his urine-until somebody empties his bladder.

The second argument against slow decompression is that it is not worth while because intravesical tension in the chronically distended bladder ceases to be significant after the first few ounces of urine have escaped. This can only hold good when the bladder is completely atonic, if the detrusor retains any contractility at all it must surely be capable of taking up some slack, however little this may be, provided too much is not asked of it at once. It must, however, be admitted that less and less is to be expected of decompression as emptying progresses.

\section{BIBLIOGRAPHY}

SCOTT, W. W. (1929);, F. Urol., $21,527$.

SHACKMAN, R., and MESSENT, D. (1954), Brit. med. F., ii, I,009.

LANE, V. J. (1955), Ұ. Irish Med. Assoc., 36, I8.

PAGE (1955), P.R.S.M., 48, 696. 